\title{
UN NOMOGRAMME \\ POUR LA DÉTERIMINATION DU DEGRÉ D'ACIDITÉ DE LA CRÈmE dÉGRAISSÉE
}

\author{
par \\ E. E. VAN DE GEHUCHTE
}

Ecole technique supérieure de l'état pour l'agriculture et les industries agricoles GAND

Au point de vue technique, afin d'obtenir des propriétés aromatiques ou devant la nécessité de traiter de la crème fermière, on utilise généralement de la crème acidifiée.

Même pour la fabrication "en continu" du beurre, qui était établie sur le traitement de la crème fraîche, on a repris le chemin classique de l'acidification de la crème.

La fermentation préalable de la crème, à cause des modifications physiques et biochimiques, exerce une grande influence sur la marche du processus de fabrication, sur la qualité du beurre et du babeurre, et sur le rendement. Il est compréhensible qu'une fabrication normale avec des produits uniformes puisse seulement être acquise quand on essaie d'obtenir de jour en jour des conditions meilleures.

La méthode graphique, que nous présentons ici, a pour but de simplifier cette standardisation et de la mettre à la portée de chaque usine. En parlant de l'influence du degré d'acidité et de la teneur en matière grasse de la crème sur la préparation du beurre, nous rappellerons en même temps les normes les plus courantes.

\section{I. - Degré d'acidité de la crème et acidité de la crème dégraissée.}

Le degré d'acidité de la crème, sans considération de la teneur en matière grasse, correspond à une notion qui induit en erreur. C'est pourquoi le degré d'acidité actuel, ou $p H$, est une grandeur plus sûre pour apprécier l'acidification de la crème. Il donne, sans dépendre de la teneur en matière grasse, une mesure directe de l'acide lactique produit. Les microorganismes ne sont pas sensibles à l'acide lactique comme tel, mais bien à la concentration d'ions hydrogène. Un désavantage, lié à la mesure du $p H$, est que les expériences techniques sont, pour la plupart, rendues en degré d'acidité titrable. De cette façon, on trouve dans la littérature peu de données et de normes basées sur le $p H$. Ce phénomène est facile à expliquer : le contrôle du $p H$ est jusqu'à maintenant limité à 
quelques laiteries, tandis que la détermination titrimétrique de l'acidité est pratiquement employée dans toutes les usines.

Une firme allemande a déjà mis au point un système continu de contrôle du $p \mathrm{H}$; lequel système, quand on atteint un certain $p \mathrm{H}$, refroidit la crème à partir d'une valeur établie. Il est certain, qu'à l'avenir, une standardisation automatique de l'acidification, avec des potentiomètres appropriés, sera plus généralement introduite dans les beurreries.

Entre temps, nous sommes toujours obligés de contrôler le degré d'acidité par la titration d'après Dornic [1]. Prenons deux échantillons de crème possédant 20 et 40 p. 100 de matière grasse et révélant tous les deux degrés un degré d'acidité de $40^{\circ} \mathrm{D}$ (fig. 1).

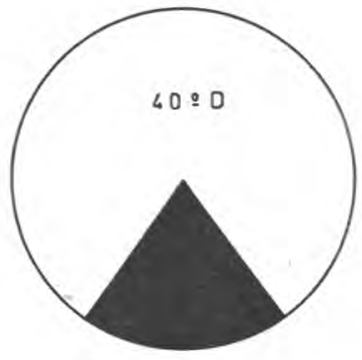

$20 \%$ matière grasse,

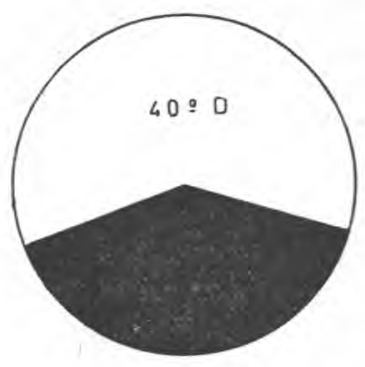

$40 \%$ matière grasse

Fig. 1.

Echantillons de crème d'un même degré d'acidité, mais différents par le taux de matière grasse (plus est élevée la teneur en matière grasse, et plus petite est la fraction d'eau contenant l'acide)

Pour l'échantillon à 20 p. 100 de matière grasse, la partie dégraissée, 80 p. 100 de la fraction de crème, correspond à $40^{\circ} \mathrm{D}$; pour l'autre, 60 p. 100 seulement de la fraction de crème contient la même quantité d'acide. Il est évident que, dans le cas présent, cette fraction est plus acide pour 40 p. 100 de matière grasse que pour 20 p. 100. Si nous ramenons le degré d'acidité à la partie dégraissée, nous obtenons :

80 p. $100 \ldots \ldots \ldots \ldots .40^{\circ} \mathrm{D}$

100 p. $100 . \frac{40 \times 100}{80}=50^{\circ} \mathrm{D}$
60 p. $100 \ldots \ldots \ldots \ldots$

100 p. $100 \frac{40 \times 100}{60}=66^{\circ} \mathrm{D}$

Seule, cette expression du degré d'acidité a, sans tenir compte de la teneur en matière grasse, une valeur comparative. Pour le rapport direct entre le degré d'acidité $\left({ }^{\circ} \mathrm{D}\right)$ de la crème contenant ( $\nabla)$ de matière grasse et le degré d'acidité de la crème dégraissée, on peut employer la relation suivante : 
Degré d'acidité de la crème dégraissée $=\frac{100 \times{ }^{\circ} \mathrm{D}}{100-\mathrm{v}}$

Cette relation est discutée plus loin (V).

Il est possible d'éviter ce calcul lorsqu'on détermine le degré d'acidité sur le sérum [2]. Etant donné que l'on contrôle quand même normalement la teneur en matière grasse, la détermination du degré d'acidité sur le sérum de la crème n'est pas à recommander, car, en plus, cette opération nécessite une longue filtration. Il existe peu de normes de cette valeur. L'action tampon des protéines est éliminée dans cette détermination de telle sorte qu'il n'y a plus de rapport direct entre le degré d'acidité du levain et celui de la crème.

\section{II. - Importance du contrôle de la teneur en matière grasse et du degré d'acidité sur le processus de barattage.}

\section{a) Technique classique (barattage).}

Le processus d'acidification est à laisser de côté, dans le cadre de la standardisation de la crème. Le déroulement de ce processus a, comme il est connu, une grande influence sur le barattage, le degré d'humidité, la consistance, les propriétés aromatiques, la conservation $d u$ beurre, et une influence moindre sur la teneur en matière grasse du babeurre.

Rappelons-nous seulement l'acidification classique à froid à température constante variant entre 12 et $15^{\circ} \mathrm{C}$ et l'acidification à chaud entre 16 et $20^{\circ} \mathrm{C}$; après 8 à 9 heures, nous obtenons un $p H$ limite de 4,5 à 5 (fig. 2), ensuite on refroidit jusque 9 à $10^{\circ} \mathrm{C}$. En hiver, on peut, éventuellement, comme pour l'acidification à froid, accepter une acidification constante jusqu'au $p H$ 4,6 à des températures de 16 à $170 \mathrm{C}$ afin de diminuer la consistance du beurre.

Le graphique 2 nous donne directement le rapport $p H$ (lignes $p \mathbf{H})$, degré d'acidité et teneur en matière grasse, ainsi que le degré d'acidité de la crème dégraissée (points sur l'ordonnée). Les lignes de $p H$ 6,8-5 et 4,6 nous donnent directement le degré d'acidité de la crème fraîche, de la crème acidifiée et de la crème mûre. Tous les degrés d'acidité des crèmes avec une teneur en matière grasse déterminée, qui se trouvent sur la même ligne de $p \mathbf{H}$, ont la même acidité dans la crème dégraissée, correspondant avec la valeur du point d'intersection de l'ordonnée et de la ligne de $p H$.

Pour des degrés d'acidité très élevés de la crème dégraissée on obtient : une perte de matière grasse due à l'englobement de la 
graisse par les protéines, une mauvaise conservation due à la teneur plus élevée de matière dégraissée dans le beurre et à l'action de catalyse de l'acidité sur les réactions chimiques qui provoquent les défauts du beurre, un goût rance du beurre, un barattage plus rapide, et, pour des degrés d'acidité trop bas de la crème dégraissée, on obtient : un mauvais barattage, des propriétés aromatiques faibles, davantage de danger de défauts du beurre dûs aux microbes, un temps de barattage plus long.

b) Préparation du beurre " en continu ».

Les données concernant le degré d'acidité et la teneur en matière grasse pour la préparation du beurre nous ont été communiquées en grande partie par Ir. Techn. De Smet, Directeur de la laiterie Deftinge, et sont basées sur les résultats d'usines belges et allemandes.

En Allemagne, on prépare le plus souvent le beurre en partant de la crème fraîche, et le type Westphalia correspond à cet effet. L'optimum de la teneur en matière grasse de la crème est 45 p. 100 - pour la crème fraîche on compte sur un degré d'acidité de $12^{\circ} \mathrm{D}$. Les difficultés commencent lors du traitement de la crème acide. Les pertes en matière grasse sont surtout importantes sur le babeurre de telle sorte que cette matière grasse doit être récupérée par centrifugation. Le degré d'acidité maximum ne pourrait être que 25 à $35^{\circ} \mathrm{D}$; il est néanmoins préférable de ne pas dépasser $20^{\circ} \mathrm{D}$.

En France, comme en Belgique, on n'est pas partisan du beurre provenant de crème fraîche. En France, le traitement du beurre de ferme dans les laiteries oblige à travailler également la crème acide ; c'est pour cette raison que le Contimab est un des premiers appareils " en continu »le mieux approprié au traitement de la crème acide.

A cette fin, il était nécessaire de corriger le système de façon qu'on ait moins de pertes sur le babeurre et qu'il soit possible de bien laver le beurre. La meilleure teneur en matière grasse de la crème est de 36 à 40 p. 100 . Les meilleurs résultats ont été obtenus par un degré d'acidité de $35^{\circ} \mathrm{D}$. Pour une acidité légère, on ne va que jusqu'à $20^{\circ} \mathrm{D}$; pour une acidité plus forte, on va jusqu'à $65^{\circ} \mathrm{D}$. Toutefois, les pertes en matière grasse qui, pour la crème légèrement acide ne dépassent pas 8 à 10 p. 100 dans le babeurre, sont ici plus élevées. Pour la crème fraîche, on a même obtenu une teneur en matière grasse de 3 p. 1000 pour le babeurre. Pour la crème acide, le pourcentage en matière dégraissée dans le beurre, peut atteindre 2,5 p. 100 , et la nécessité de bien laver le beurre est évidente. 
Westphalia, avec son type à deux cylindres, nous permet également de travailler de la crème acide. En Allemagne, on trouve superflu le lavage du beurre tant que la crème reste au-dessous de $40^{\circ} \mathrm{D}$. L'optimum de la teneur en matière grasse est de 35 à 40 p. 100 , et celui du degré d'acidité de 35 à $40^{\circ} \mathrm{D}$. Une acidité plus élevée n'est pas un inconvénient.

Encore plus d'importance est portée au lavage du beurre dans le type Roth à trois cylindres - optimum de la teneur en matière grasse : 36 p. 100 - le degré d'acidité peut atteindre 54-570 D.

\section{TABLEAU 1}

CARACTÉRISTIQUES DE STANDARDISATION DE LA CREMME POUR LES PROGÉDÉS " EN GONTINU " D'APRES FRITZ

\begin{tabular}{|c|c|c|c|c|c|}
\hline Type & $\begin{array}{c}\text { Teneur } \\
\text { en matière } \\
\text { grasse } \\
\text { de la crème }\end{array}$ & $\begin{array}{c}\text { Degré } \\
\text { d'acidité } \\
\text { de Ia crème }\end{array}$ & $\begin{array}{c}\text { Degré } \\
\text { d'acidité } \\
\text { de la crème } \\
\text { dégraissée }\end{array}$ & $\begin{array}{l}\text { Température } \\
\text { de barratage }\end{array}$ & $\begin{array}{r}\text { Perte en \% } \\
\text { de matière } \\
\text { grasse dans } \\
\text { le babeurre }\end{array}$ \\
\hline WESPHALIA & 45 & $\begin{array}{c}\text { fraîche: } \\
12^{\circ} \mathrm{D} \\
\text { acide : } \\
20^{\circ} \mathrm{D}\end{array}$ & $\begin{array}{l}22^{\circ} \mathrm{D} \\
36^{\circ} \mathrm{D}\end{array}$ & $\begin{array}{c}\text { été : } \\
6-8^{\circ} \mathrm{C} \\
\text { hiver : } \\
12-13^{\circ} \mathrm{C}\end{array}$ & $1,2 \cdot 1,5$ \\
\hline Contimab & $36-40$ & $\begin{array}{c}\text { Légèrement } \\
\text { acide : } \\
20^{\circ} \mathrm{D} \\
\text { moyen : } \\
30-35^{\circ} \mathrm{D} \\
\text { fort : } \\
55-60^{\circ} \mathrm{D}\end{array}$ & $\begin{array}{c}\text { jusqu'à } \\
32^{\circ} \mathrm{D} \\
49-56^{\circ} \mathrm{D} \\
\text { jusque } \\
90^{\circ} \mathrm{D}\end{array}$ & $\begin{array}{c}\text { été : } \\
9-10^{\circ} \mathrm{C} \\
\text { hiver : } \\
12-13^{\circ} \mathrm{C}\end{array}$ & $\begin{array}{c}\text { été : } \\
0,8-0,9 \\
\text { hiver : } \\
0,3-0,5 \\
\text { été : } \\
1,1-1,2 \\
\text { hiver } \\
0,8-0,9 \\
\text { plus de } 1,0\end{array}$ \\
\hline $\begin{array}{c}\text { WESPHALIA } \\
\text { type à } \\
2 \text { cylindres }\end{array}$ & $35-40$ & $35-40^{\circ} \mathrm{D}$ & $\begin{array}{c}56-64^{\circ} \mathrm{D} \\
1\end{array}$ & $\begin{array}{c}\text { été : } \\
9-10^{\circ} \mathrm{C} \\
\text { hiver : } \\
12-13^{\circ} \mathrm{C}\end{array}$ & $\begin{array}{c}\text { été : } \\
0,4-0,8 \\
\text { hiver : } \\
0,3-0,5\end{array}$ \\
\hline $\begin{array}{c}\text { Rотн } \\
\text { type à } \\
3 \text { cylindres }\end{array}$ & $30-36$ & $54.57^{\circ} \mathrm{D}$ & $77-82^{\circ} \mathrm{D}$ & $\begin{array}{c}\text { été : } \\
9-10^{\circ} \mathrm{C} \\
\text { hiver : } \\
12-13^{\circ} \mathrm{C}\end{array}$ & $\begin{array}{c}\text { été : } \\
0,5-0,6 \\
\text { hiver : } \\
0,3-0,4\end{array}$ \\
\hline
\end{tabular}


De là, on conclut qu'avec les derniers types, le maniement de la crème correspond de plus en plus à celui de la maturation ordinaire de la crème et que la crème fermentée, possédant un degré d'acidité de $78^{\circ} \mathrm{D}$, peut également y être travaillée.

Le tableau 1 nous donne les caractéristiques essentielles des différents procédés Fritz, et l'évolution citée nous apparaît encore mieux.

L'influence sur les pertes en matière grasse n'est pas seulement due aux types de machines, à la teneur en matière grasse, au degré d'acidité et à la température de la crème, mais également à l'ajustage, au réglage de la machine et au traitement préalable de la crème.

Nous pouvons accepter comme règle générale que, pour une teneur en matière grasse plus élevée de la crème, la teneur en matière grasse du babeurre est également plus élevée sans que pour cela les pertes soient plus considérables.

\section{III. - Influence du degré d'acidité de la crème dégraissée sur les propriétés aromatiques.}

Nous avons déjà démontré que la croissance et les propriétés biochimiques des microorganismes dépendent de $(\mathbf{H}+)$. Ceci est, en effet, le cas pour les organismes acidifiants. Le levain est un mélange équilibré de Streptococcus lactis ou cremoris et de Leuconostoc citrovorus. Les premières espèces sont les producteurs de l'acide lactique et les dernières, du produit aromatique diacétyl. Les bactéries aromatiques ne se développent seulement que lorsque les bactéries acidifiantes ont créé un degré d'acidité approprié et également lorsque les conditions de croissances se sont améliorées par la démolition des protéines. Le développement des bactéries aromatiques commence normalement à un $p H \quad 5,5$ ou à un degré d'acidité de la crème dégraissée d'environ $35^{\circ} \mathrm{D}$, ce développement et la formation des produits aromatiques sont les plus grands dans la crème mûre pour un $p H$ 4,5-4,6 [5].

Quoique la température optimum des bactéries aromatiques soit de $37^{\circ} \mathrm{C}$, cette température n'est pas la plus favorable pour la formation de produits aromatiques. On obtient les meilleurs résultats lorsque la crème est acidifiée jusqu'à $46^{\circ} \mathrm{D}$, à une température de 17 à $20^{\circ} \mathrm{C}$, ensuite refroidie et acidifiée à une température de 8 à $10^{\circ} \mathrm{C}$ [4]. Une longue conservation à un degré d'acidité élevé est nuisible vu que pour une croissance trop développée des bactéries aromatiques, celles-ci montrent une action réductrice et transforment le diacetyl en acétylmethylcarbinol et butylène- 
glycol, qui sont inodores. Est à considérer comme limite l'acidification jusqu'à $p H$ 4,6 correspondant à un degré d'acidité dans la crème dégraissée de $78^{\circ} \mathrm{D}$ (fig. 2).

Les résultats changeants dans l'acidification et la formation de produits aromatiques, qui sont trop facilement attribués aux cultures, trouvent souvent leur origine dans les conditions divergentes qui existent dans les usines et qui sont négligées par faute de contrôle.

\section{IV. - Détermination du degré d'acidité.}

En Belgique, comme en France, l'acidité de la crème est généralement déterminée d'après la méthode Dornic. A cet effet : mesurer au moyen d'une pipette $10 \mathrm{ml}$ de crème et titrer en présence de $0,5 \mathrm{ml}$ de phénolphtaléine avec $\mathrm{NaOH}$ N/9 jusqu'au virage rose pâle. Rincer la pipette à crème au moyen de la crème neutralisée et ajuster le mélange avec $\mathrm{NaOH}$ jusqu'au virage rose pâle. Un dixième de $\mathrm{ml}$ de solution alcaline correspond à un degré Dornic $\left({ }^{\circ} \mathrm{D}\right)$ de la crème.

Comme la masse spécifique de la crème dépend de la teneur en matière grasse et qu'en même temps le pipetage d'une crème grasse peut produire quelques difficultés, il est à conseiller d'employer une méthode gravimétrique pour la détermination exacte du degré total d'acidité. La teneur en matière grasse déterminée par méthode butyrométrique est également exprimée gravimétriquement.

En place de $10 \mathrm{ml}$ la titration est exprimée sur $10 \mathrm{~g}$.

On pèse la crème jusqu'à deux décimales après la virgule, en prenant soin d'avoir une quantité de crème qui contient approximativement $10+1 \mathrm{~g}$.

Afin de spécifier l'élément gravimétrique de cette détermination de l'acidité nous symbolisons l'acidité par oDg [6].

L'acidité ${ }^{\circ} \mathrm{Dg}$ est donc égale à la quantité de dixième de $\mathrm{ml}$ de solution alcaline $\mathrm{N} / 9$ nécessaire pour neutraliser $10 \mathrm{~g}$ de crème.

\section{V. - Détermination du degré d'acidité de la crème dé- graissée.}

Lors de la discussion du degré d'acidité de la crème dégraissée, nous avons vu qu'on pouvait obtenir cette valeur en employant la relation $\frac{100 \times{ }^{\circ D g} \text {. }}{100-v}$ 
Au cas où le poids n'est pas exactement de $10 \mathrm{~g}$, il est possible de rapporter cette relation à $10 \mathrm{~g}$ exactement, comme suit :

$\mathrm{oDg}$ crème dégraissée $=\frac{100 \times 0 \mathrm{Dg}}{100-\mathrm{v}} \times \frac{10}{\mathrm{~g}}(1)$

Pour éviter de confondre le degré d'acidité de la crème et celui de la crème dégraissée, nous représentons le degré d'acidité de la crème par $\alpha$ et celui de la crème dégraissée par oDg, ainsi nous obtenons dans (1):

$$
\begin{aligned}
{ }^{\circ} \mathrm{Dg} & =\frac{1.000 \times \alpha}{(100-v) \mathrm{g}}(2) \\
\text { ou, pour } \mathrm{g}=10,{ }^{\circ} \mathrm{Dg} & =\frac{100 \times \alpha}{100-\mathrm{v}}(3)
\end{aligned}
$$

Il est possible de représenter ces relations par un Nomogramme (7) (fig. 3).

Le but du Nomogramme est de déterminer facilement, sans calcul, la valeur o $\mathrm{Dg}$, connaissant le nombre de dixièmes de $\mathrm{ml}$ de solution alcaline employée pour neutraliser g gramme de crème.

Les trois lignes verticales de la figure 3 représentent respectivement de droite à gauche :

$1^{\circ}$ La teneur en matière grasse de la crème exprimée en p. 100 du poids jusqu'à 0,1 p. 100 ;

$2^{\circ}$ L'acidité de la crème est égale à la valeur $\alpha$ au cas où juste $10 \mathrm{~g}$ de crème ont été pesés.

$\alpha=$ le nombre de dixièmes de $\mathrm{ml}$ de solution alcaline employée pour $10 \mathrm{~g}$ de crème ou le nombre de $\mathrm{ml}$ de solution alcaline employée pour $100 \mathrm{~g}$ de crème, de façon que l'expression " acidité de la crème » soit justifiée.

$\mathrm{Au}$ cas où la quantité de crème pesée n'est pas exactement $10 \mathrm{~g}$, il est nécessaire de ramener la valeur $\alpha$ à $10 \mathrm{~g}$ de crème. Cette correction est exposée plus loin.

$3^{\circ}$ L'acidité de la crème dégraissée exprimée en ${ }^{\circ} \mathrm{Dg}$ quand l'acidité de la crème a été déterminée au moyen d'une solution alcaline $\mathrm{N} / 9$.

$\mathrm{Vu}$ la rareté d'un poids exact de $10 \mathrm{~g}$ l'emploi de Nomogramme simple (que représente la relation (2) est pratiquement impossible. Dans ce cas on peut ramener la formule (3) à (2) en employant la verticale à l'extrême gauche (échelle de correction de poids).

$$
\text { Exemple: } \begin{aligned}
\mathrm{g} & =10,90 \mathrm{~g} \\
\alpha & =60,0 \mathrm{ml}
\end{aligned}
$$




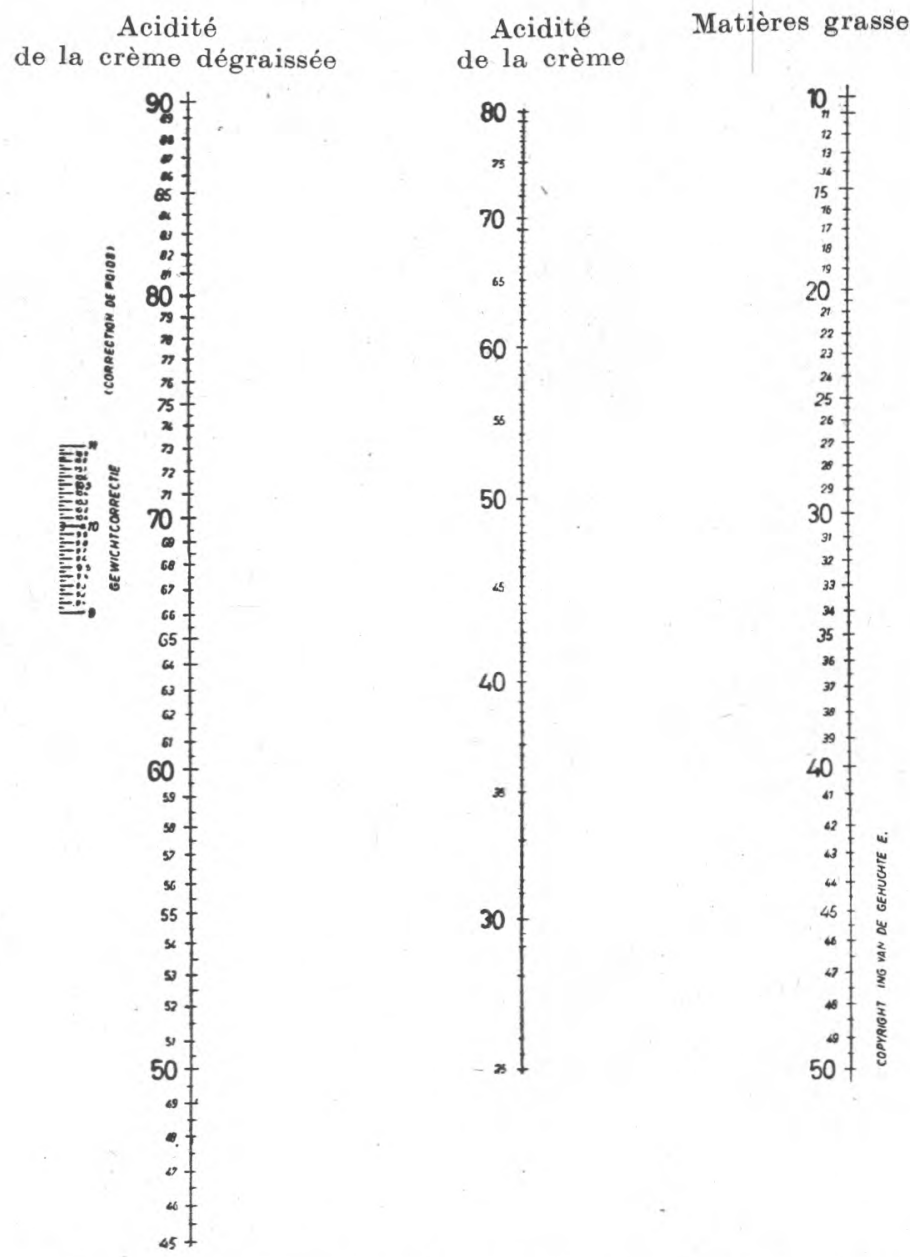

Fig. 3. - Nomogramme pour la détermination du degré d'acidité de la crème dégraissée, partant de la teneur en matière grasse et du degré d'acidité de la crème.

Plaçons l'échelle de correction de poids contre l'échelle de l'acidité de la crème de façon que 10,9 correspondent à 60,0 on obtient en face de la petite flèche la valeur corrigée de l'acidité de la crème $(55)$ correspondant à $10 \mathrm{~g}$ de crème.

La figure 4 donne l'exemple d'un calcul à l'aide du Nomogramme.

\section{VI. - Standardisation du babeurre (8).}

Nous voulons encore ajouter une méthode de contrôle simple du babeurre pour la standardisation de la crème. 
de la crème dégraissée

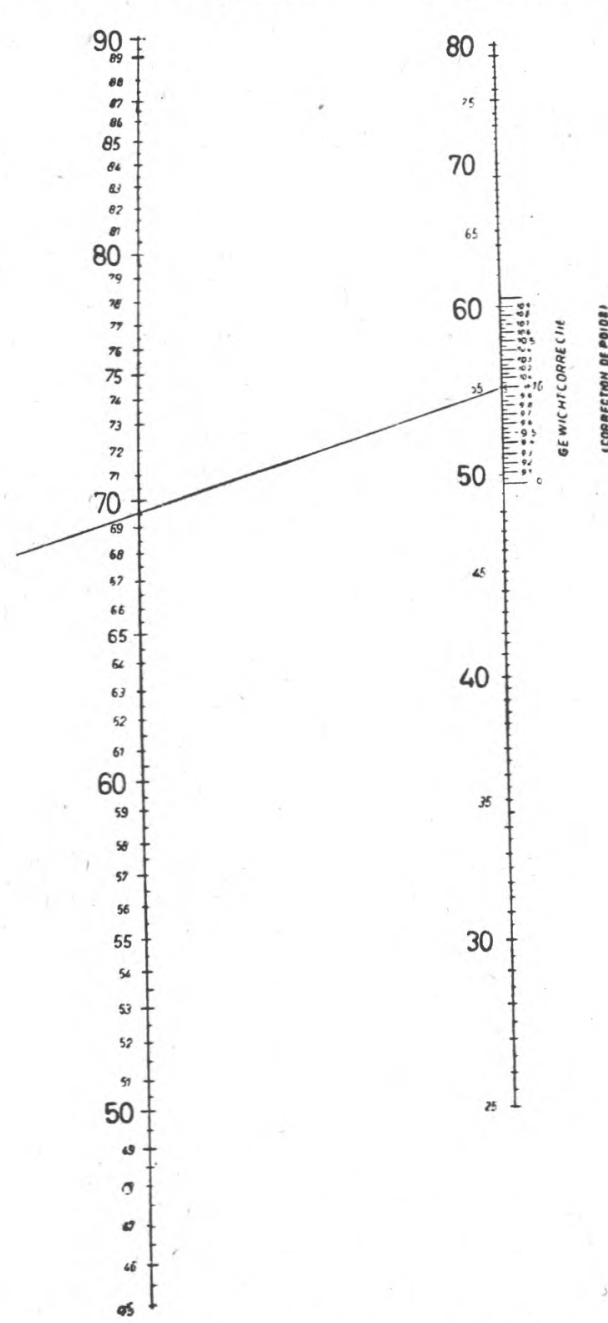

Matières grasses \%

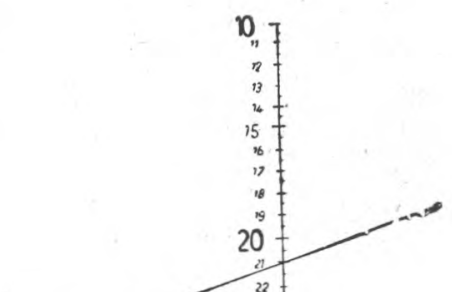

Fig. 4. - Nomogramme pour la détermination du degré d'acidité de la crème dégraissée, partant de la teneur en matière grasse et du degré d'acidité de la crème.

Le communiqué ministériel du 19 janvier 1932 prescrit que pour la Belgique le babeurre doit contenir 6,8 p. 100 de matière sèche dégraissée. Les normes néerlandaises NN 1616 prévoient la dérivation de la matière sèche à partir de la teneur en matière grasse et de la densité. Pour la pratique belge, s'impose le problème : 


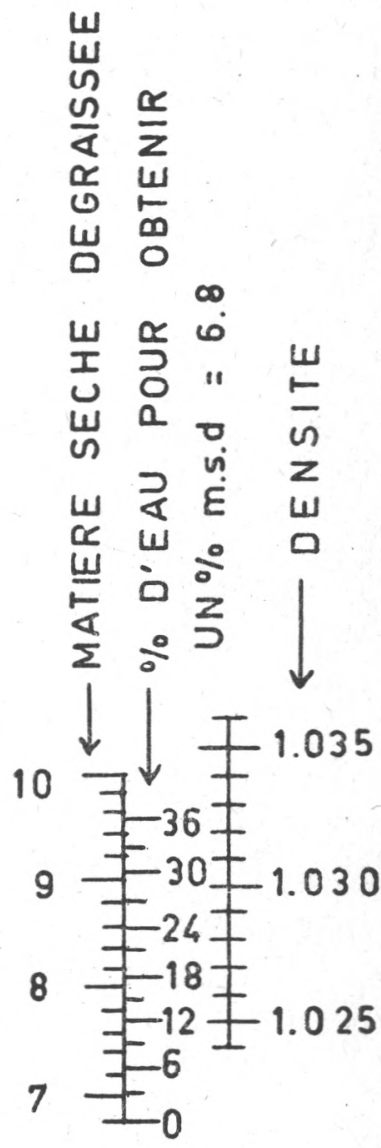

$u$
$\frac{1}{\alpha}$
$\frac{w}{5}$
$\Sigma$

$\stackrel{0}{\circ}$

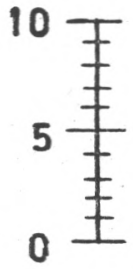


quand le babeurre a une densité $d x$ et une teneur en matière grasse $\nabla x$, combien d'eau peut-on ajouter afin que le babeurre reste dans les limites de la loi. Il est possible de résoudre facilement ce problème en se basant sur les formules de la matière sèche :

$$
(\mathrm{D}-\mathrm{v})=\mathrm{r}=0,23 \mathrm{v}+2,6 \times \frac{[100(\mathrm{~d} 20-0,9982)]}{\mathrm{d} 20}
$$

et pour les valeurs possibles de $\mathrm{v}(\mathrm{v} x)$, de déterminer les valeurs d20 $(\mathrm{dx})$ de façon que $\mathrm{r}$ soit supérieur à 6,8 .

Cette solution a un inconvénient : pour le contrôle, $r$ est déterminé par la méthode scientifique $(\mathrm{D}-\mathrm{v})$. Les difficultés se présentent seulement pour les cas limites et lors d'un ajustage précis du babeurre sur une matière sèche dégraissée de 6,8 p. 100 .

En employant le Nomogramme (fig. 5) on détermine d'une manière simple combien de litres d'eau on peut encore ajouter à 100 litres de babeurre et quelle valeur de matière sèche dégraissée on obtient. A cet effet, on relie par une droite la valeur trouvée de la teneur en matière grasse du babeurre sur l'échelle de graisse (échelle inférieure à l'extrême gauche) et la valeur trouvée de la densité sur l'échelle de densité (échelle supérieure à l'extrême droite), puis on détermine le point d'intersection sur l'échelle du milieu. Sur cette échelle on lit alors :

- A gauche : la matière sèche dégraissée du babeurre;

- A droite : la quantité d'eau en litres qui peut être ajoutée à 100 litres de babeurre afin d'obtenir du babeurre de 6,8 p. 100 . de matière sèche dégraissée.

En France, où aucune norme n'est prévue, il est possible de calculer au moyen du Nomogramme combien de matière sèche dégraissée est contenue dans le babeurre en partant de la graisse et de la densité.

\section{RÉSUMÉ}

Le degré d'acidité et la teneur en matière grasse de la crème sont à interpréter ensemble lorsqu'on fait le contrôle de la préparation du beurre.

Lorsqu'on exprime le degré d'acidité sur la crème dégraissée on obtient des valeurs comparatives pour des teneurs en matière grasse différentes. L'importance de la standardisation de la crème pour la préparation du beurre est donnée pour la préparation avec la baratte et la préparation continue du beurre.

Pour l'exécution du contrôle, on a rappelé qu'en Belgique comme en France, on employait la méthode Dornic pour déterminer l'acidité. On propose de travailler sur $10 \mathrm{~g}$ au lieu de $10 \mathrm{ml}$ et l'aci- 
dité serait rapportée à la crème dégraissée (acidité symbolisée par oDg).

Un Nomogramme a été exécuté pour la relation :

$$
{ }^{\circ} \mathrm{Dg}=\frac{10}{\mathrm{~g}} \times \frac{100 \times \alpha}{100-\nabla}
$$

où $\mathrm{g}=$ le poids pesé de crème,

$\mathrm{v}=$ p. 100 de teneur en matière grasse,

$\alpha=$ quantité de dixièmes de $\mathrm{ml}$ de solution alcaline $\mathrm{N} / 9$.

L'expression 10/g est représentée par une échelle «correction de poids "; la suite de la relation est représentée par un diagramme simple.

Il est enfin établi, par une méthode graphique, comment on peut calculer la matière sèche dégraissée du babeurre au moyen de la teneur en matière grasse et de la densité.

\section{SUMMARY}

When expressing the degree of acidity on degreased cream, comparative values are obtained for different contents of fatty matter.

It is proposed to perform titration on $10 \mathrm{~g}$ instead $10 \mathrm{ml}$ and the acidity would be added to the degreased cream (symbol of acidity : $\mathrm{oDg}$ ).

A nomogram has been executed for the relation

$$
{ }^{\circ} \mathrm{Dg}=\frac{10}{\mathrm{~g}} \times \frac{100 \times \alpha}{100-v}
$$

where $\mathrm{g}=$ the weight weighed of cream,

$\mathrm{v}=$ content $\mathrm{p} .100$ in fatty matter,

$\alpha=$ quantity of tenths of ml alkalin solution $\mathrm{N} / 9$.

The expression $10 \mathrm{~g}$ is represented by a " weight correction scale ", the rest of the relation is represented by a simple diagram. It is finally established by a graphic method, how to calculate the dry degreased matter of the buttermilk by means of the content in fatty matter and density.

\section{BIBLIOGRAPHIE}

[1] E. E. VAN DE GehUChte : Zuivelgids, p. 256 (Vyncke-Gent).

[2] E. E. VAN DE GehUChтE : Zuivelgids, p. 262 (Vyncke-Gent).

[3] NAUDTS: Zuivelgids, p. 422 (Vyñcke-Gent). 
[4] Монв : Deut. Molkerei Zeitung, 1937, 58, 782.

[5] Pien : Le lait, 1949, 29, 461.

[6] E. E. VAN DE GeHUChtE : Zuivelgids, p. 257 (Vyncke-Gent).

[7] E. E. VAN De Gehuchte : Zuivelgids, p. 260-261 (Vyncke-Gent).

[8] E. E. VAN DE GehUChTE : Zuivelgids, p. 290 (Vyncke-Gent).

\section{SUPPLEMENT TECHNIQUE}

\section{LE PAIEMENT DU LAIT \\ D'APRÈS SA TENEUR EN PROTÉINES ET LE DOSAGE DE CETTE DERNIËRE}

par

G. GÉNIN

Ingénieur E.P.C.I.

On a reconnu depuis déjà longtemps qu'il est profondément anormal de payer le lait, bien entendu non seulement d'après son poids, mais essentiellement d'après sa teneur en graisse alors que dans beaucoup de cas ce lait est transformé en produits qui, tels que le fromage, contiennent essentiellement les graisses et les protéines du lait sous une forme concentrée.

$\mathrm{Si}$, pendant de nombreuses années, cette situation s'est maintenue, ce n'est pas par le désir de conserver une façon de faire défectueuse, mais plutôt par suite du fait qu'on ne disposait pas de méthode simple, rapide et précise permettant d'effectuer un contrôle journalier et portant sur des centaines d'échantillons, de la teneur en protéines ou de l'extrait sec dégraissé du lait.

C'est devant l'importance toujours plus grande que l'on reconnaît aujourd'hui aux protéines du lait dans la nourriture de l'homme que les chercheurs de laboratoire se sont attachés à la question et sont parvenus à mettre au point des méthodes plus rapides et cependant suffisamment précises pour permettre la détermination de la teneur en protéines du lait.

Il n'est pas étonnant que l'application de ces méthodes se soit rapidement généralisée dans les pays importants producteurs de fromage, comme par exemple les Pays-Bas, et deux grandes Associations coopératives laitières ont dès à présent adopté ces méthodes permettant le paiement du lait en fonction de sa teneur en protéines. 